\title{
Raman Microspectroscopy and Its Role in Solving Today's Food Industry Challenges
}

\author{
Steven Zbylut $^{1}$
}

1. Dr. Steven Zbylut, General Mills International/Medallion Laboratories, Minneapolis, USA.

This presentation will focus on the advantages of Raman microspectroscopy and its applicability for use in solving food industry challenges ${ }^{[1-4]}$. The benefits of Raman spectroscopy are numerous and include the ability to non-destructively analyze samples in-situ, without any interference from water, while obtaining results in real-time without modification of the sample. Traditional microscopy utilizes stains to help identify protein, fat or carbohydrates in food samples; however, Raman microspectroscopy allows the user to not alter the sample or the ingredients location while still being able to detect and identify each of these ingredients at the same time. The inherent advantage of Raman microspectroscopy is that one can characterize the vibrational modes of all the ingredients being used in the product by evaluating neat samples first, and then looking for conserved vibrational modes in the complex finished food product itself. A great example of the viability of this technique is that certain ingredients that cannot be stained for, such as flavor compounds or additives, can still be identified within the sample since the spectroscopic data can be analyzed by looking for key vibrational modes that are characteristic of these ingredients.

In order to investigate the applicability of this technology, a study was designed to isolate and identify the location of a specific flavor compound in a suitable food matrix such as chewing gum. In order to evaluate these polymer systems, Raman spectra were acquired of all of the neat compounds typically found in a gum base matrix. Once these compounds had been evaluated, Raman microspectroscopy analyses were then performed on the final gum base matrix in order to better understand feasibility of this technique to identify the key compounds being utilized. These initial Raman maps, typically 100 micrometer x 200 micrometer in size, were acquired by rastering a $780 \mathrm{~nm}$ laser in 2 micrometer steps across the surface of the sample at a collection rate of 10 seconds per step. This process would generate a Raman map of the surface that could then be further analyzed to determine the exact location of key ingredients by utilizing the Raman marker bands that had been previously identified from the neat Raman spectra (see Figure 1 below). Once feasibility had been determined, gum base samples with unique flavor emulsions were then evaluated in-situ to clearly locate the flavor compounds of interest (see Figure 2).

Results were able to demonstrate how the distribution of flavor emulsions could be monitored in-situ in a gum base system which allowed for certain patents to be submitted and granted (US20140295024, WO2012116152, EP2677878, CA2825543, AU2012220602B, RU2552059) based off of the findings from this new and enabling technology.

In conclusion, the versatility of this methodology is just starting to be realized due to the advancements in laser technology, detection systems, mapping capabilities and software advancements that allow for collecting and interrogating rather large sample areas in extremely short times. A thorough discussion of Raman microspectroscopy and its advantages will be presented along with examples of how this technology has been used to evaluate gum and food based systems. 
References:

[1] Burt, D.J., Fearn, T., Starch 35 (1983), p. 351.

[2] Piot, O., Autran, J.C. and Manfait, M., Journal of Cereal Science 34 (2001), p. 191.

[3] Pudney, P.D., Hancewicz, T.M., Cunningham, D.G., Spectroscopy 16 (2002), p. 217.

[4] Pudney, P.D., Gambelli, L., Gidley, M.J., Applied Spectroscopy 65 (2011), p. 127.

Color Intensity is related to location and intensity in the order of Red $>$ Yellow $>$ Green>Blue
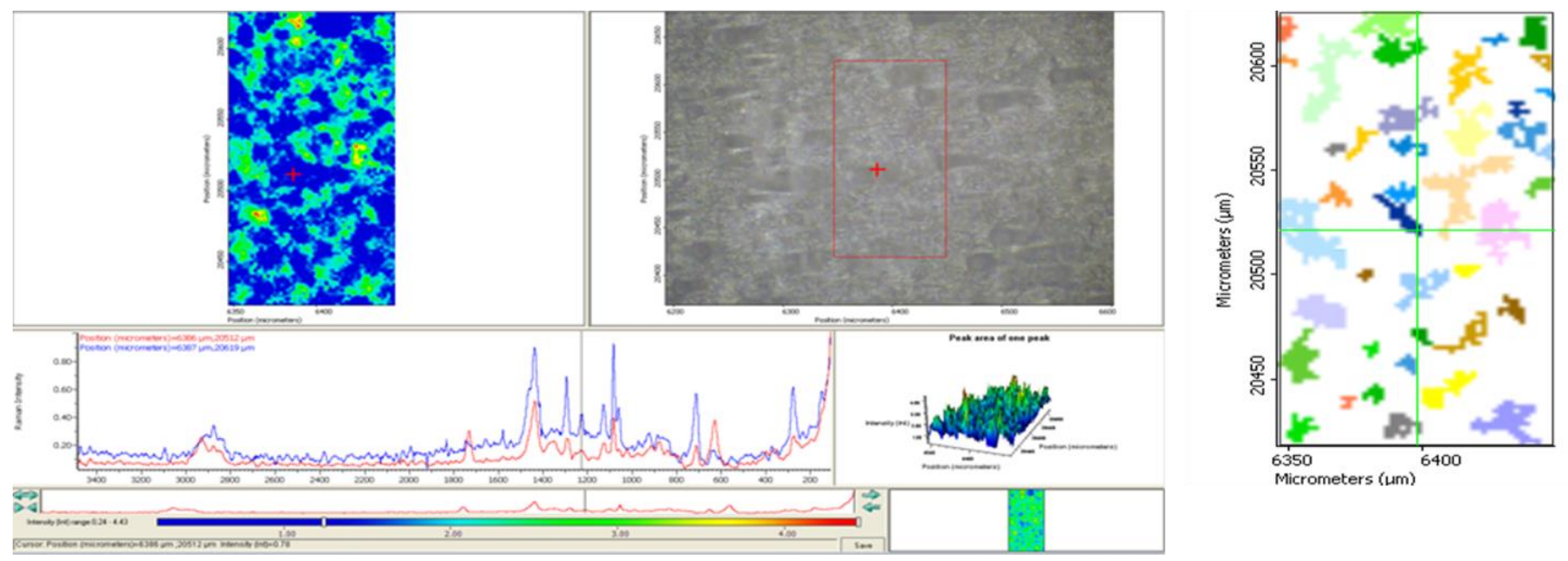

Figure 1. Distribution of Oil/Wax in Gum Base by utilizing the $1292 \mathrm{~cm}^{-1}$ Raman marker band. 2 micrometer x 2 micrometer steps were acquired across the 100 micrometer x 200 micrometer sample area which resulted in the acquisition of 5408 total spectra.

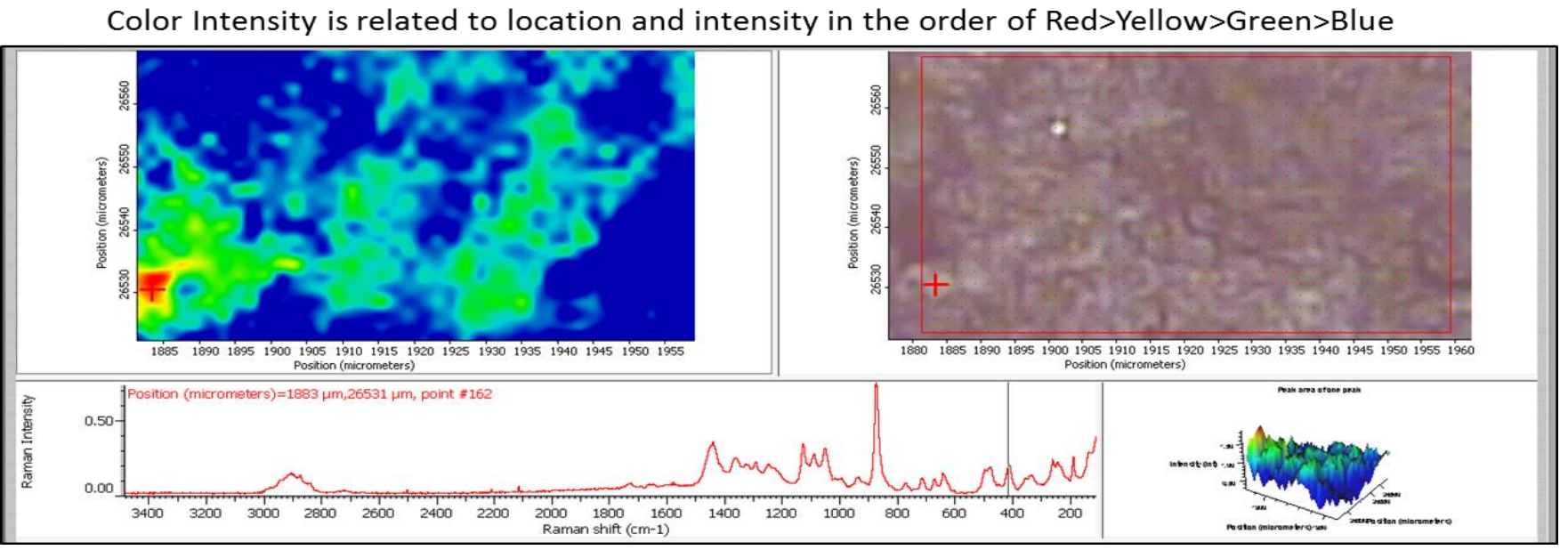

Figure 2. Distribution of Flavor Emulsion by utilizing the $408 \mathrm{~cm}^{-1}$ Raman marker band. 2 micrometer x 2 micrometer steps were acquired across the 80 micrometer x 50 micrometer sample area which resulted in the acquisition of 960 total spectra. 